\title{
Apple, Alphabet, or Microsoft: Which Is the Most Efficient Share?
}

\author{
Paulo Ferreira* \\ *CEFAGE-UE, IIFA, Universidade de Évora \\ Submitted: April 28, $2016 \bullet$ Accepted: July 6, 2016
}

\begin{abstract}
Studying the efficiency of financial assets is important because, if they are not efficient, this means that investors have some capacity to predict the behavior of those assets. In this paper, we use detrended fluctuation analysis to assess the efficiency of the three most valuable American companies, which, curiously, all happen to be from the same economic sector: Apple, Alphabet, and Microsoft. The results point to the efficiency of Apple's shares and to similar results regarding Alphabet. Only Microsoft shares show evidence of deviations from efficiency. Our results also suggest that moments of crisis could have an impact on the efficiency pattern of shares.
\end{abstract}

JEL classification: F36, G2, G21, G34, L1

Keywords: detrended fluctuation analysis, return rates, efficiency, Apple, Alphabet, Microsoft

\section{Introduction}

According to the Financial Times' Global 500 (FT 500), at the end of 2015, Apple, Alphabet (formerly Google), and Microsoft were the three firms with the highest market capitalization of all the listed companies. Curiously, all three companies work in the information technology and communication sector, and are constantly striving to develop innovative products and services.

\footnotetext{
${ }^{*}$ Corresponding Author. Email: pjsf@uevora.pt
} 
Staying on top of the FT 500 is important for these companies, but also for current and potential investors, because it is synonymous with the shares' health and, of course, with the potential dividend and profit gains should those shares be sold. Consequently, it is important to analyze the behavior of these shares. To pursue this objective, we will analyze the efficiency of the aforementioned shares. According to the Efficient Market Hypothesis in its weak form, proposed by Malkiel and Fama (1970), the returns of financial assets have no memory. In other words, return rates should not have any kind of dependence in their time series.

The first studies of the Efficient Market Hypothesis were performed analyzing linear autocorrelations, and generally concluded that this dependence does not exist or, if it does, it is not significant in the short-term (Bonanno et al., 2001). However, besides linear autocorrelations, other nonlinear dependences could persist in return rates. This objective has raised physicists' interest in the financial markets, using methodologies from statistical physics. One of those methodologies is the detrended fluctuation analysis (DFA), which makes it possible to detect long-range dependences in a given time series. If, as expected, return rates have no memory, the corresponding asset prices will behave like a random walk process. If, on the other hand, the series indicates some kind of dependence, this could be a sign of inefficiency, although it is necessary to prove whether such a divergence from a random walk would indicate any capability of predicting return rates.

In this paper, we will use DFA to analyze the behavior of Apple, Alphabet, and Microsoft shares and the efficiency of these stocks. We will apply DFA to the whole series' return rates and then apply the same methodology to different partitions after analyzing the existence of structural breaks. Our main results point to an efficient pattern by Apple's shares, though there was some deviation in the last of four subperiods (defined by the structural break test); to a lesser degree, Alphabet's shares also behaved in what could be considered a near-efficient manner. Only Microsoft's shares had some evidence of inefficiency. These results should be analyzed more deeply in order to confirm whether it would be possible for investors to predict the behavior of these stocks.

The remainder of this paper is organized as follows. Section 1 briefly summarizes the Efficient Market Hypothesis (EMH), which is mainly focused on nonlinear approaches. Section 2 presents the data we analyzed and DFA, section 3 shows the results, and section five concludes and discusses those results.

\section{Brief Analysis of the Efficient Market Hypothesis}

The existing literature includes several studies about financial markets dedicated to analyzing dependence and, consequently, efficiency. One of the first analyses of such issues was Bachelier's (1900), which analyzed the probability of the distribution of stock prices and confirmed 
its normality (as expected). The random, independent and identical distribution of return rates was corroborated by subsequent studies, such as Kendall and Hill (1953), Osborne (1964), and Granger and Morgenstern (1964), among others, which confirmed the feature of random walk in financial markets and their lack of memory. These publications had in common the analysis of linear autocorrelations, a feature that has been continually confirmed by recent studies (e.g., Ferreira and Dionísio, 2014, 2016). The behavior of financial series as a random walk is present in several models and hypotheses of financial markets, including the EMH proposed by Malkiel and Fama (1970). However, in addition to these studies, some authors found that financial markets suffer from several stylized facts-characteristics of financial markets that occur frequently. Works such as those of Cont (2001) or Parisi et al. (2013) summarize a number of those stylized facts, such as the existence of fat tails in returns, asymmetries in gains and losses, volatility clustering, leverage effect, correlation between trading volumes and volatility, and autocorrelation in the variance. Although it is not possible for these studies to prove that these anomalies have an effect on the capability of predicting return rates, it is important to analyze the dependence of stock markets and, consequently, their efficiency. The fact is that, if return rates have memory, it could be possible to predict the behavior of assets and, as such, to find violations of the EMH.

An interesting feature of studies that found stylized effects is the fact that they frequently used nonlinear approaches to study financial markets. The starting point of these studies is the fact that, even when the absence of linear autocorrelation is not rejected, markets could be inefficient due to nonlinear dependences (e.g., Darbellay, 1998; Granger et al., 2004). Some studies do, however, use nonlinear approaches. For example, Mandelbrot (1977) analyzed stock markets using fractional statistics and found evidence of fractal dimensions, which indicates long-term dependence. Studies like those of Barkoulas and Baum (1996), Sadique and Silvapulle (2001), Di Matteo et al. (2005), and Christodoulou-Volos and Siokis (2006), among many others, also found evidence of long-range dependence in financial markets.

One of the specific methodologies used to study dependence in time series is DFA. Based on the Hurst exponent (Hurst, 1951), DFA was created by Peng et al. (1994) to study the behavior of DNA. This methodology was then extended to other research areas, including financial markets. For example, Liu et al. (1997) found persistent volatility in the S\&P 500, and Ausloos et al. (1999) and Ausloos (2000) found evidence of long-term dependence in exchange markets. In terms of studies specifically examining stock markets - and using only more recent examples - Ferreira and Dionísio (2014), Kristoufek (2015), Cao and Zhang (2015), and Anagnostidis et al. (2016) also found evidence of dependence. It should be noted, however, that the main objective of our paper is not an in-depth literature review of EMH once we analyze the specific behavior of Apple, Alphabet, and Microsoft shares; for a more complete survey of EMH, see Sewell (2011). 


\section{Data and Methodology}

In this study we analyze the behavior of share returns for Apple, Alphabet, and Microsoft by market capitalization. We retrieved data from the date each company was listed until April 22, 2016; the dates are different for each company but, as our objective was to compare the behavior of each series individually, this is not an issue. We retrieved the information on share prices from Datastream, then calculated the return rates for those series after eliminating the information of non-trading days due to holidays from our sample. Considering the price level $P_{t}$, we calculated the return rates using the difference of consecutive logarithms, e.g., $r_{t}=\ln \left(P_{t}\right)-\ln \left(P_{t-1}\right)$.

Our proposal was to study these three companies' stock efficiency, analyzing the temporal dependence in their returns using DFA. The main objective of this technique was to analyze the relationship between values $x_{t}$ and $x_{t+s}$ at different points in time. Considering a time series $x_{k}$, with $k=1, \ldots, N$ equidistant observations, DFA starts integrating the original time series, obtaining $x(t)=\sum_{k=1}^{t} x_{k}$. Then, this new series is divided in $N / s$ mutually exclusive boxes of equal dimension, $s$. The methodology goes on to obtain the trend, $z_{s}(t)$, of each segment with ordinary least squares and calculate $x_{s}(t)=x(t)-z_{s}(t)$, which is a detrended series. In the original application, a linear trend is assumed, which we also used in this paper. Later applications showed the possibility of including other polynomial trends (Kantelhardt et al., 2001). Once we had obtained the values of $x_{s}(t)$ and $z_{s}(t)$ for each

box, we then calculated the DFA function, given by $F(s)=\sqrt{\frac{1}{N} \sum_{t=1}^{N}\left[x_{s}(t)\right]^{2}}$. Averaging this value for all centered boxes in $s$, we generated the value of $\langle\mathrm{F}(\mathrm{s})\rangle$, a function of $s$. We repeated that calculation for all different values of $s$, and then expected a power-law behavior of $\langle F(s)\rangle \sim s^{\alpha}$.

The information about efficiency is given by the $\alpha$ parameter, which is used to analyze serial dependence. If $\alpha=0.5$, that time series is represented by a random walk, which means that it no longer has memory. However, if $0.5<\alpha<1$ then the series has a positive longrange dependence (the time series is persistent, which means that if returns are positive in a given period, it is more likely that they will continue to be positive in the next period); if $\alpha<0.5$, the series has a negative long-range dependence (it is antipersistent - if returns had been up in the previous period, they are more likely to be down in the next period, or viceversa). According to this theory, it is expected that return rates would display the behavior of a random walk. Although DFA gives us information about the efficiency of a given stock, it is possible that, over time, the degree of efficiency may change. We considered the possibility of our series having structural breaks and, as such, we performed Bai and Perron's structural break test (1998; 2003a; 2003b), which allowed us to identify multiple unknown breakpoints. This test had advantages when compared to previous tests: the Chow (1960) test implied 
a priori knowledge about the date of the break, although the Quandt (1960) test played down the necessity of this requirement. With the knowledge of those structural breaks, we evaluated the evolution of efficiency in each company's stock over time, again using DFA.

\section{Results}

First, we performed a brief analysis of our data, which can be found in Figure 1. The behavior of the shares' prices is shown on the left, and the behavior of their respective return rate on the right. As demonstrated at the bottom of the figure, the three shares had a sustained increase in price over time: Apple's daily return rates' average is about $0.06 \%$, Microsoft's about $0.08 \%$ and Alphabet's about $0.09 \%$. This table also shows that volatility, measured by the standard deviation, was similar for all three shares. Finally, the level of kurtosis found in all shares was larger than the expected values for a normal distribution. This result is coherent with the existence of fat tails, a much-documented stylized effect in financial literature.

After these preliminary results, we performed DFA on the whole sample, the results of which are presented in Table 2. These results show that Apple's shares could be considered efficient: the DFA exponent is almost equal to the expected 0.5 value. The remaining two shares - Alphabet and Microsoft - both have lower DFA exponent values, which means that their stocks show anti-persistent behavior. However, these results should be interpreted in different ways. In addition to the difference between Alphabet's DFA exponent and 0.5, the absolute difference is smaller than the one observed in Microsoft, which shows a larger difference from the expected value and could be a sign of this stock's inefficiency.

In order to analyze the quality of the results of the DFA exponent, we made a log- $\log$ graph for each share (Figure 2), which shows that the r-square of the regression is always very high, implying that the DFA exponent estimation is very good.

Regardless of Alphabet having a comparatively smaller data sample than Apple and Microsoft, with more than a decade of data, we have large samples from which to draw our conclusions. In this case, it would be advisable to test the possible existence of structural breaks. Using the Bai and Perron structural break test, we analyzed the three prices' time series, the results of which are presented in Tables 3, 4, and 5. From this we concluded that Apple has three structural breaks (implying that we can divide the whole sample into four subsamples), whereas Alphabet and Microsoft each have four structural breaks (and, as such, five subsamples). It is also interesting to note that, with the exception of one case between Apple and Alphabet, the dates of the structural breaks among the three shares do not coincide.

Our objective when dividing each sample into subsamples was to analyze, for each share, 
the behavior of efficiency over time. So, we again applied DFA to each subsample, and have presented those results in Table 6. Due to space constraints, we have not presented the graphs to demonstrate the quality of the exponents, but the fitting process is, again, of high quality. These figures will be provided upon request.

Apple's results show that its shares exhibited a behavior near efficiency until the fourth subsample: in the first three subsamples, the DFA exponent is near 0.5; however, the last value allows us to conclude that Apple's shares have an anti-persistent behavior. This last subsample starts in October 2010.

Three subsamples of Alphabet's shares show results that are coherent with efficiency: the first, second, and fourth. The remaining two subsamples have evidence of anti-persistence: the third subsample lasted from July 2008 until October 2010 and the fifth started in October 2013.

Finally, Microsoft's results show that, regardless of the subsample in question, its shares were far from the efficiency level. Furthermore, the exponent shows an anti-persistence pattern across all subsamples, which means that if returns are positive in a given period, it is more likely that they'll be negative in the next one, or vice-versa. Another interesting feature is the fact that the latest subsamples show significant anti-persistence for all three series.

\section{Conclusions}

Since the third quarter of 2015, Apple, Alphabet, and Microsoft are the most valuable companies in the United States. This fact is not only very important to the companies themselves, but also to investors. As such, studying the efficiency of these companies' shares is important because the results could give investors information about such shares. In order to analyze the shares' behavior, we performed DFA, an analysis technique that studies the existence of long-range dependence in a given time series and allows the evaluation of the series memory. According to the EMH, return rates will not have memory and DFA will confirm this.

First, we applied DFA to the samples retrieved as a whole. Our results show that Apple could be considered an efficient share and that the behavior of Alphabet's shares could also be deemed efficient. On the other hand, Microsoft shows evidence of anti-persistence, which could be seen as a sign of inefficiency to some extent. Next, we performed the Bai and Perron structural break test to detect multiple structural breaks in the original series. With the results of that test, we divided each sample into subsamples, and found that Apple and Alphabet had results that were consistent with efficiency. Nevertheless, we detected some instances where results fell far away from the efficiency result. Contrary to those shares, Microsoft consistently showed evidence of anti-persistence in its series, which could be interpreted as inefficiency. 
These results are interesting for current and potential investors because inefficiency could imply the existence of a prediction capability in the series. However, these results should be interpreted with caution, because it has not been proven that deviations from a random walk pattern imply profitability for such shares. Further studies on these assets should be conducted to evaluate this possibility. Another interesting feature of the results comes from examining the structural breaks. Some of the detected breaks are related to crisis moments; Apple's last structural break, for example, is in October 2010, when the sovereign Euro crisis started. Although this largely affected a different continent, the contagion effect could have had some influence on Apple's stock prices. Alphabet also had a structural break in October 2010; furthermore, this particular share had another structural break in July 2008, near of the occurrence of the subprime crisis. Although these two moments are not relevant to any of Microsoft's structural breaks, its last break is near the 9/11 terrorist attacks; despite not being an economic crisis, this could have had an impact on the behavior of Microsoft's stock.

These results, along with the fact that the latest subsamples show evidence of antipersistence, could mean that the existence of crisis or negative events could have an effect on the efficiency pattern of a given asset.

\section{References}

Anagnostidis, P., Varsakelis, C., and Emmanouilides, C. (2016). Has the 2008 financial crisis affected stock market efficiency? The case of Eurozone. Physica A: Statistical Mechanics and its Applications, 447:116 - 128.

Ausloos, M. (2000). Statistical physics in foreign exchange currency and stock markets. Physica A: Statistical Mechanics and its Applications, 285(1 - 2):48 - 65.

Ausloos, M., Vandewalle, N., Boveroux, P., Minguet, A., and Ivanova, K. (1999). Applications of statistical physics to economic and financial topics. Physica A: Statistical Mechanics and its Applications, 274(1 - 2):229 - 240 .

Bachelier, L. (1900). Théorie de la spéculation. Annales scientifiques de l'École Normale Supérieure, 3(17):21 - 86.

Bai, J. and Perron, P. (1998). Estimating and Testing Linear Models with Multiple Structural Changes. Econometrica, 66(1):47 - 78 .

Bai, J. and Perron, P. (2003a). Computation and analysis of multiple structural change models. Journal of Applied Econometrics, 18(1):1 - 22. 
Bai, J. and Perron, P. (2003b). Critical values for multiple structural change tests. Econometrics Journal, 6(1):72 - 78 .

Barkoulas, J. T. and Baum, C. F. (1996). Long-term dependence in stock returns. Economics Letters, 53(3):253 - 259 .

Bonanno, G., Lillo, F., and Mantegna, R. N. (2001). Levels of complexity in financial markets. Physica A: Statistical Mechanics and its Applications, 299(1-2):16 - 27.

Cao, G. and Zhang, M. (2015). Extreme values in the Chinese and American stock markets based on detrended fluctuation analysis. Physica A: Statistical Mechanics and its Applications, 436:25 - 35.

Chow, G. C. (1960). Tests of Equality Between Sets of Coefficients in Two Linear Regressions. Econometrica, 28(3):591 - 605.

Christodoulou-Volos, C. and Siokis, F. M. (2006). Long range dependence in stock market returns. Applied Financial Economics, 16(18):1331 - 1338.

Cont, R. (2001). Empirical properties of asset returns: stylized facts and statistical issues. Quantitative Finance, 1(2):223 - 236.

Darbellay, G. A. (1998). Predictability: An Information-Theoretic Perspective. In Procházka, A., Uhlír, J., Rayner, P. W. J., and Kingsbury, N. G., editors, Signal Analysis and Prediction, pages 249 - 262. Birkhäuser Boston, Boston, MA.

Di Matteo, T., Aste, T., and Dacorogna, M. M. (2005). Long-term memories of developed and emerging markets: Using the scaling analysis to characterize their stage of development. Journal of Banking and Finance, 29(4):827 - 851. Risk Measurement.

Ferreira, P. and Dionísio, A. (2014). Revisiting serial dependence in the stock markets of the G7 countries, Portugal, Spain and Greece. Applied Financial Economics, 24(5):319 - 331.

Ferreira, P. and Dionísio, A. (2016). How long is the memory of the US stock market? Physica A: Statistical Mechanics and its Applications, 451:502 - 506.

Granger, C. W., Maasoumi, E., and Racine, J. (2004). A Dependence Metric for Possibly Nonlinear Processes. Journal of Time Series Analysis, 25(5):649 - 669.

Granger, C. W. J. and Morgenstern, O. (1964). Spectral analysis of New York stock market prices. In Cootner, P., editor, The random character of stock market prices, pages 162 188. Cambridge, MA: MIT Press. 
Hurst, H. E. (1951). Long-term storage capacity of reservoirs. Transactions of the American Society of Civil Engineers, 116:770 - 808.

Kantelhardt, J. W., Koscielny-Bunde, E., Rego, H. H., Havlin, S., and Bunde, A. (2001). Detecting long-range correlations with detrended fluctuation analysis. Physica A: Statistical Mechanics and its Applications, 295(3 - 4):441 - 454.

Kendall, M. G. and Hill, A. B. (1953). The Analysis of Economic Time-Series-Part I: Prices. Journal of the Royal Statistical Society. Series A (General), 116(1):11 - 34.

Kristoufek, L. (2015). Detrended fluctuation analysis as a regression framework: Estimating dependence at different scales. Physical Review E, 91:022802.

Liu, Y., Cizeau, P., Meyer, M., Peng, C.-K., and Stanley, H. E. (1997). Correlations in economic time series. Physica A: Statistical Mechanics and its Applications, 245(3):437 440.

Malkiel, B. G. and Fama, E. F. (1970). Efficient Capital Markets: A Review of Theory and Empirical Work. The Journal of Finance, 25(2):383 - 417.

Mandelbrot, B. (1977). The fractal geometry of nature. New York, NY: W. H. Freeman and Company.

Osborne, M. F. M. (1964). Brownian motion in the stock market. In Cootner, P., editor, The random character of stock market prices, pages 262 - 296. Cambridge, MA: MIT Press.

Parisi, D. R., Sornette, D., and Helbing, D. (2013). Financial price dynamics and pedestrian counterflows: A comparison of statistical stylized facts. Physical Review E, 87:012804.

Peng, C.-K., Buldyrev, S. V., Havlin, S., Simons, M., Stanley, H. E., and Goldberger, A. L. (1994). Mosaic organization of DNA nucleotides. Physical Review E, 49:1685 - 1689.

Quandt, R. E. (1960). Tests of the Hypothesis That a Linear Regression System Obeys Two Separate Regimes. Journal of the American Statistical Association, 55(290):324 - 330.

Sadique, S. and Silvapulle, P. (2001). Long-term memory in stock market returns: international evidence. International Journal of Finance \& Economics, 6(1):59 - 67.

Sewell, M. (2011). History of the efficient market hypothesis. Research Note No. RN/11/04, London, UK: UCL Department of Computer Science. 
Figure 1: Graphical description of prices and return rates, and descriptive statistics for Apple, Alphabet, and Microsoft shares
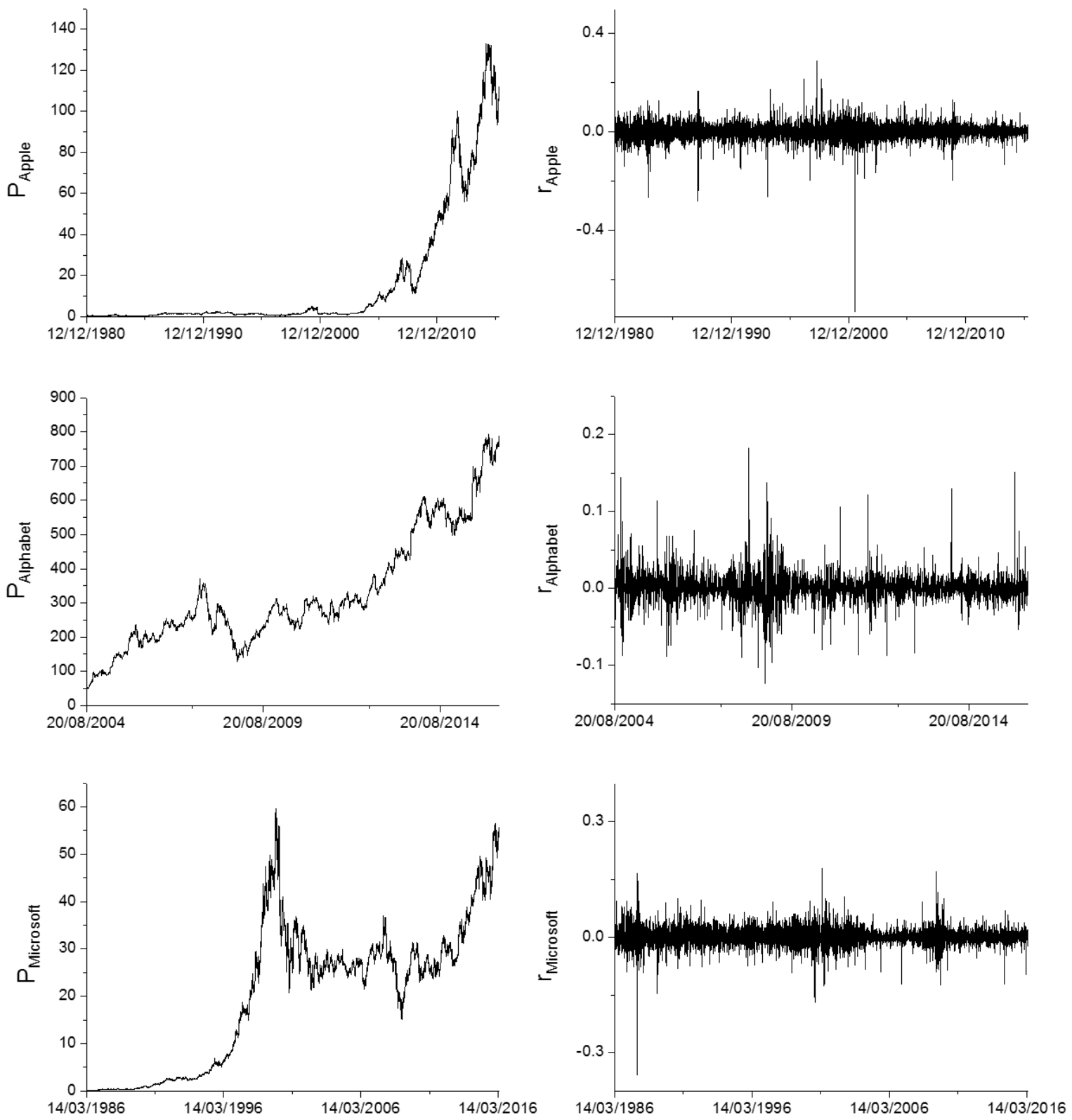

\begin{tabular}{lccc}
\hline & Apple & Alphabet & Microsoft \\
\hline Mean & $0,0599 \%$ & $0,0934 \%$ & $0,0839 \%$ \\
Std. Dev. & 0,0302 & 0,0200 & 0,0222 \\
Kurtosis & 44,8992 & 9,3293 & 14,8660 \\
\hline
\end{tabular}


Figure 2: Quality of the DFA exponents

\section{Apple}

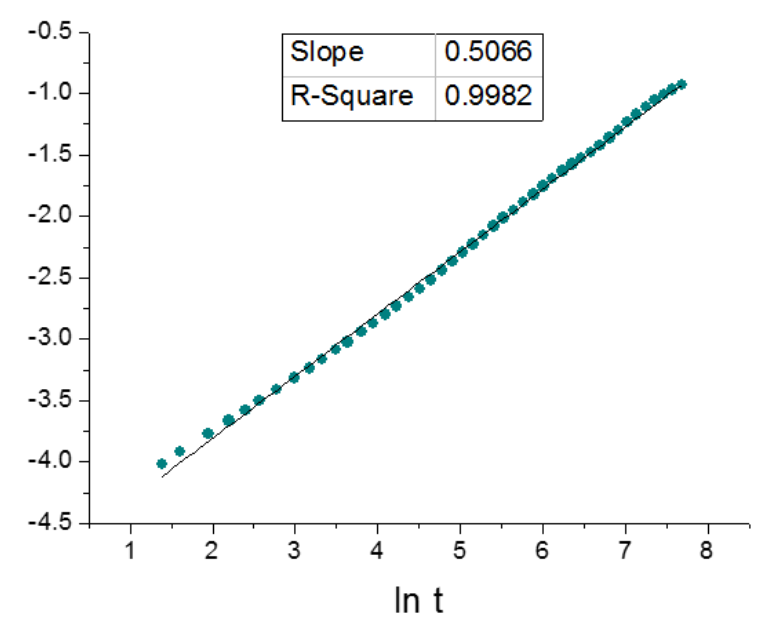

\section{Alphabet}

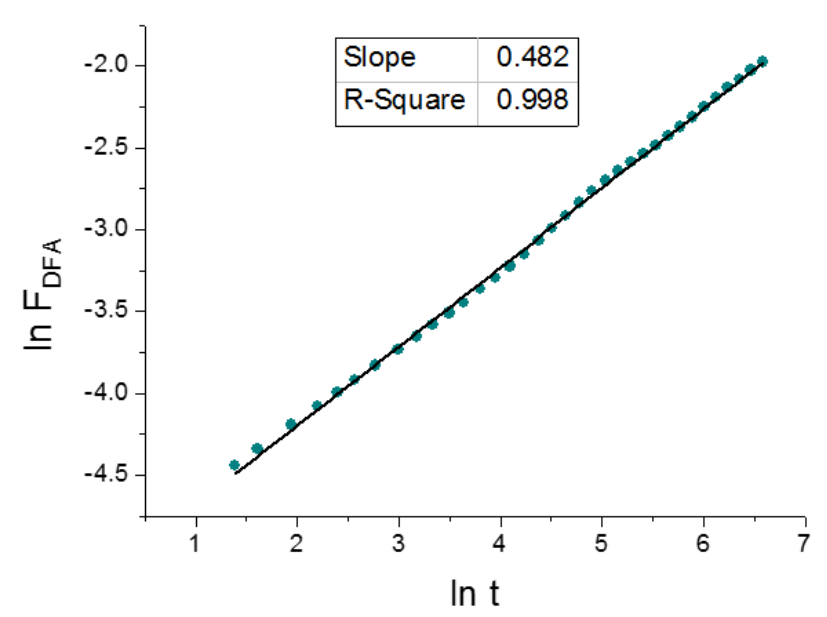

\section{Microsoft}

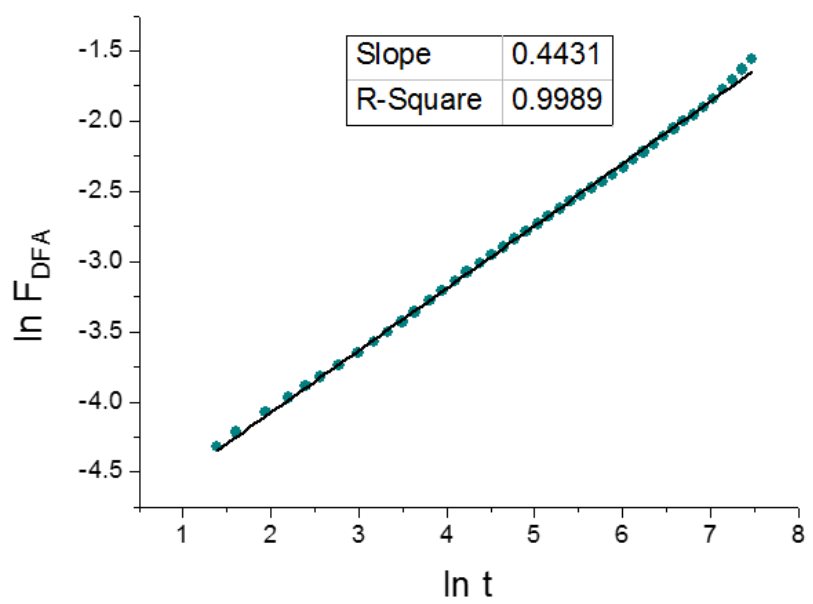


Table 1: Sample Information

\begin{tabular}{lcc}
\hline Share & Date & $N$ \\
\hline Apple & $12 / 12 / 1980$ & 8889 \\
Alphabet & $19 / 08 / 2004$ & 2939 \\
Microsoft & $13 / 03 / 1986$ & 7575 \\
\hline
\end{tabular}

Table 2: DFA Results for the Complete Sample of Shares

\begin{tabular}{lc}
\hline Share & DFA Exponent \\
\hline Apple & $0.5067 \pm 0.0033$ \\
Alphabet & $0.4829 \pm 0.0029$ \\
Microsoft & $0.4432 \pm 0.0023$ \\
\hline
\end{tabular}

Table 3: Results of the Bai and Perron Structural Break Test for Apple

\begin{tabular}{lrr}
\hline Break Test & F-Statistic & Break Date \\
\hline 0 vs 1 & $47,296.7^{*}$ & 4665 (July 7, 1999) \\
1 vs 2 & $3,089.5^{*}$ & 6169 (June 30, 2005) \\
2 vs 3 & $16.4^{*}$ & 7558 (October 15, 2010) \\
3 vs 4 & 7.2 & \\
\hline
\end{tabular}

Note: *denotes rejection of null hypothesis with a significance value of $5 \%$. 
Table 4: Results of the Bai and Perron Structural Break Test for Alphabet

\begin{tabular}{lrr}
\hline Break Test & F-Statistic & Break Date \\
\hline 0 vs 1 & $9,657.9^{*}$ & 537 (October 6, 2006) \\
1 vs 2 & $1,328.2^{*}$ & 986 (July 21, 2008) \\
2 vs 3 & $489.7^{*}$ & 1550 (October 14, 2010) \\
3 vs 4 & $83.8^{*}$ & 2309 (October 21, 2013) \\
4 vs 5 & 0.0 & \\
\hline
\end{tabular}

Note: *denotes rejection of null hypothesis with a significance value of $5 \%$.

Table 5: Results of the Bai and Perron Structural Break Test for Microsoft

\begin{tabular}{lrr}
\hline Break Test & F-Statistic & Break Date \\
\hline 0 vs 1 & $28,889.5^{*}$ & 1851 (July 26, 1993) \\
1 vs 2 & $1,969.9^{*}$ & 2987 (January 28, 1998) \\
2 vs 3 & $1,285.2^{*}$ & 4123 (August 7, 2002) \\
3 vs 4 & $961.8^{*}$ & 6440 (October 18, 2011) \\
4 vs 5 & 0.9 & \\
\hline
\end{tabular}

Note: *denotes rejection of null hypothesis with a significance value of $5 \%$.

Table 6: DFA Results for the Different Subsamples

\begin{tabular}{cccccc}
\hline & Apple & \multicolumn{2}{c}{ Alphabet } & & Microsoft \\
\hline Sub & DFA Exponent & Sub & DFA Exponent & Sub & DFA Exponent \\
\hline 1 & $0.4895 \pm 0.0038$ & 1 & $0.4991 \pm 0.0020$ & 1 & $0.4546 \pm 0.0030$ \\
2 & $0.4986 \pm 0.0051$ & 2 & $0.5050 \pm 0.0169$ & 2 & $0.3863 \pm 0.0026$ \\
3 & $0.5249 \pm 0.0095$ & 3 & $0.4039 \pm 0.0029$ & 3 & $0.4659 \pm 0.0073$ \\
4 & $0.4677 \pm 0.0067$ & 4 & $0.4871 \pm 0.0043$ & 4 & $0.4503 \pm 0.0064$ \\
& & 5 & $0.4309 \pm 0.0049$ & 5 & $0.4296 \pm 0.0057$ \\
\hline
\end{tabular}

